"Quasi una tragedia delle attioni humane » : le tragique entre allégorie et édification morale dans l'œuvre de Fabio Glissenti (1542-1615)

"Quasi una tragedia delle attioni humane»: il tragico fra allegoria ed edificazione morale nell'opera di Fabio Glissenti (1542-1615)

"Quasi una tragedia delle attioni humane": Tragic between Allegory and Moral Edification in Fabio Glissenti’s Works (1542-1615)

\title{
Eugenio Refini
}

\section{OpenEdition Journals}

Édition électronique

URL : http://journals.openedition.org/cei/2214

DOI : $10.4000 /$ cei.2214

ISSN : 2260-779X

Éditeur

UGA Éditions/Université Grenoble Alpes

Édition imprimée

Date de publication : 1 novembre 2014

Pagination : 185-198

ISBN : 978-2-84310-285-1

ISSN : $1770-9571$

Référence électronique

Eugenio Refini, « «Quasi una tragedia delle attioni humane » : le tragique entre allégorie et édification morale dans l'œuvre de Fabio Glissenti (1542-1615) », Cahiers d'études italiennes [En ligne], 19 | 2014, mis en ligne le 01 mai 2016, consulté le 27 mars 2021. URL : http://journals.openedition.org/cei/2214 ; DOI : https://doi.org/10.4000/cei.2214 


\title{
«QUASI UNA TRAGEDIA DELLE ATTIONI HUMANE » : LE TRAGIQUE ENTRE ALLÉGORIE ET ÉDIFICATION MORALE DANS L'OEUVRE DE FABIO GLISSENTI (1542-1615)
}

\author{
Eugenio Refini \\ Johns Hopkins University - Baltimore
}

L'allégorie et l'édification morale comptent parmi les composantes les plus importantes du théâtre spirituel entre le Moyen Âge et l'époque moderne. Bien que voué au salut des âmes chrétiennes - voué donc à un dénouement heureux - le théâtre spirituel trouve néanmoins dans la notion de "tragédie» un autre élément d'une certaine importance. C'est en fait à travers un véritable processus de purification (une sorte de catharsis chrétienne) que les personnages et - surtout - le public de spectateurs et de lecteurs reconnaissent leurs propres péchés ${ }^{1}$. Également, c'est à travers le remords de sa conscience que le public peut gagner le salut éternel. Dans le contexte de la Renaissance tardive et de l'âge baroque, cette idée de tragédie spirituelle trouve un terrain fécond non seulement dans le domaine proprement dramatique, mais aussi dans la grande variété des usages métaphoriques de l'imaginaire théâtral, qui est — comme Curtius l'avait déjà très bien montré - un des éléments caractéristiques de la période ${ }^{2}$.

Une seule image éloquente pour commencer : une devise tiré d'un recueil d'emblèmes sacrés publié à Vérone par le médecin, philosophe et écrivain Francesco Pona en $1645^{3}$. Le titre, Cardiomorphoseos, qui s'inspire

I. Pour la notion de catharsis, voir notamment Aristote, Poétique, 6, I449 b 25-30.

2. Voir E. R. Curtius, Letteratura europea e Medio Evo Latino, Florence, La Nuova Italia, 1995, p. I58 et suiv.; en ce qui concerne la métaphore du theatrum mundi, qui sera centrale dans les pages qui suivent, voir également R. Bernheimer, "Theatrum mundi", Art Bulletin, n' 38, 1956, p. 225-247; M. Costanzo, Il Gran Theatro del Mondo, Milan, Scheiwiller, 1964; F. A. Yates, Theatre of the World, Londres, Routledge \& Kegan Paul, 1969 (traduction italienne: Theatrum orbis, Turin, Aragno, 2002); L. Christian, Theatrum Mundi: The History of an Idea, New York-Londres, Garland, 1987.

3. F. Pona, Cardiomorphoseos sive ex corde desumpta emblemata sacra, Vérone, Bartolomeo Merlo, 1645. Pour une introduction aux œuvres de Pona et à son profil, S. Buccini, Francesco Pona: l'ozio lecito della scrittura, Florence, Leo S. Olschki, 20I3. 
bien évidemment des Métamorphoses d'Ovide, nous dit que le recueil est consacré aux "formes» du cœur humain ("ex corde desumpta emblemata sacra»). Le premier emblème (fig. I) est une version ouvertement théâtrale du topos classique de la psychomachie, où l'on voit le cœur — image de l'âme humaine - délivré des chaînes du vice, prêt à monter vers Dieu, comme le texte de la devise le dit: "Liber En ad Te Redeo", "finalement, délivré, je reviens à toi » ${ }^{4}$. La composante dramatique de cette psychomachie visualisée est développée par le poème latin qui suit, consacré au combat allégorique entre le Cœur, la Chair, le Monde et le Diables. Tout le livre de Francesco Pona est conçu comme un itinéraire dramatique vers Dieu, un voyage pour le lecteur qui est censé s'engager dans un véritable exercice spirituel ${ }^{6}$.

L'interaction entre l'imaginaire métaphorique théâtral et les pratiques spirituelles qui étaient à l'ordre du jour à l'époque de la Contre-Réforme - bien représentée par l'emblème de Pona - est aussi au centre de la production littéraire de l'auteur sur lequel nous allons concentrer notre attention dans les pages qui suivent. Fabio Glissenti, médecin et maitre en philosophie naturelle, né à Brescia en $\mathrm{I} 542$, fit ses études à l'université de Padoue et, plus tard, s'établit à Venise en qualité de médecin auprès des Grands Hôpitaux de la ville ${ }^{7}$. À côté de quelques publications érudites — il faut au moins rappeler ici les commentaires aux œuvres logiques d'Aristote, Porphyre et Gilbert de Poitiers ${ }^{8}$ — Glissenti est l'auteur d'une série de textes littéraires curieux, si non proprement bizarres : tout d'abord un traité volumineux en forme de dialogue intitulé Discorsi morali, publié à Venise en $1596^{9}$; par la suite, à partir de 1607 , un corpus de pièces théâtrales allégoriques visant à l'édification morale des orphelines qui logeaient

4. Pona, Cardiomorphoseos, ouvr. cité, p. I.

5. Le poème latin est intitulé Peccator ad se reversus. Rhythmus Apodos. Pour l'usage des personnifications, voir au moins quelques lignes : "Errabam coecus, devius, / Septus hinc inde sordibus. / Mancipium hostis inferi, / Catenis vinctus horridis. // Forti (heu nimis) imperio, / Caro iam Carni insederat: / Mundusque iam seduxerat / Palantem (vafer) animum. // Retia saepe Diaboli/ Subivit desiderium:/ Foedo Voluntas aucupi/ Praedam se dedit miseram." (Ibid., p. I-2)

6. Pour une autre images ouvertement théâtrale, voir Pona, Cardiomorphoseos, ouvr. cité, p. 2I.

7. Un profil biographique exhaustif dans A. L. Saso, "Glissenti, Fabio», Dizionario Biografico degli Italiani, $\mathrm{n}^{\circ} 57,2001$, p. 406-408.

8. F. Glissenti, In quinque praedicabilia Porphyrij. In sex principia Gilberti Porretani. In Praedicamenta Aristotelis. In perihermenias Aristotelis. In priora, et posteriora Aristotelis. Per methodicas Divisiones brevissima commentaria, Venise, Giovan Battista Ciotti, 1594.

9. F. Glissenti, Discorsi morali dell'eccellente signor Fabio Glissenti. Contra il dispiacer del morire. Detto Athanatophilia, Venise, Domenico Farri, 1596 (une deuxième édition parut encore à Venise en I6oo, imprimée par Bartolomeo Alberti). Sur les Discorsi morali la bibliographie n'est pas riche. Voir G. McClure, "The Artes and the Ars moriendi in Late Renaissance Venice: The Professions in Fabio Glissenti's Discorsi morali contra il dispiacer del morire, detto Athanatophilia (I596)", Renaissance Quarterly, n 5I, 1998, p. 92-I27; L. Piantoni, "Morte a Venezia. L'Athanatophilia di Fabio Glissenti, I596", dans Visibile teologia. Il libro sacro figurato in 
dans les Hôpitaux où Glissenti travaillait ${ }^{\mathrm{I}}{ }^{\mathrm{D}}$. Il s'agit, en fait, d'un projet cohérent, car les pièces développent en forme dramatique la matière des Discorsi ; le dialogue, d'autre part, a un fort potentiel théâtral qui — comme nous allons le voir - nous offre une clé de lecture pour toute l'œuvre du médecin.

La structure et le but des Discorsi morali sont explicités par le frontispice (fig. 2) : "Discorsi morali contra il dispiacer del morire, detto Athanatophilia, divisi in cinque dialoghi, occorsi in cinque giornate. Ne' quali si discorre quanto ragionevolmente si dovrebbe desiderar la Morte; e come naturalmente la si vada fuggendo." Un dialogue, donc, en cinq journées, qui — selon la tradition des artes moriendi — vise à montrer que la mort ne doit pas être méprisée, mais, au contraire, désirée avec ardeur ${ }^{\mathrm{II}}$. Le frontispice souligne deux autres composantes du traité : en premier lieu une série de contes entrelacés dans la narration principale ("Con trenta vaghi e utili ragionamenti, come tante piacevoli novelle interposti»); deuxièmement la composante visuelle du livre ("adornati di bellissime figure a' loro luoghi appropriate»). En lisant les Discorsi, en fait, le lecteur se trouve face à un corpus d'images qui porte littéralement sur scène le dialogue. On rencontre, par exemple, les deux interlocuteurs principaux, le Philosophe et le Courtisan (symboles, respectivement, de la vie contemplative et de la vie active) en train de discuter, dans une mise en scène tout à fait particulière. Ils se rencontrent sur la place Saint-Marc (fig. 3) et, par la suite, il se déplacent dans Venise en esquissant un itinéraire qu'on peut suivre dans le détail : nous les voyons naviguer sur le Grand Canal, traverser le pont du Rialto ou encore bavarder sur les Fondamenta Nuove avec les îles de Saint-Michel et Murano en arrière-plan ${ }^{12}$. Le leitmotiv de ces images est la présence obsédante de la Mort : des squelettes apparaissent partout, d'après le modèle offert par la Danse Macabre de Hans Holbein,

Italia tra Cinque e Seicento, études réunies par E. Ardissino et E. Selmi, Rome, Edizioni di Storia e Letteratura, 2OI2, p. $22 \mathrm{I}-25 \mathrm{O}$.

Io. Voir, en ordre chronologique, La Ragione sprezzata, Serravalle, Marco Claseri, I6o6; Il bacio della Giustitia e della Pace, Venise, Giovanni Alberti, I607; L'Andrio cioè l'Huomo virile, Venise, Giovanni Alberti, I607; Il Diligente overo il Sollecito, Venise, Giovanni Alberti, I608; La Morte inamorata, Venise, Giovanni Alberti, I608; L'Androtoo cioè l'Huomo innocente, Venise, Marco Ginammi, I6ı; La giusta Morte, Venise, Marco Ginammi, I617; Lo Spensierato fatto pensoroso, Venise, Marco Ginammi, I6I7; Il mercato overo la fiera della vita humana, Venise, Marco Ginammi, I620; La Sarcodinamia cioè la possanza della carne, Venise, Marco Ginammi, I620.

II. Pour la tradition des artes moriendi, voir L. P. Kurtz, The Dance of Death and the Macabre Spirit in European Literature, New York, Columbia University, 1934; A. Tenenti, Il senso della morte e l'amore della vita nel Rinascimento, Turin, Einaudi, I977; sur le même sujet, qui a notamment une importance centrale dans la culture allemande à la Renaissance, voir également A. Reinis, Reforming the Art of Dying: The Ars moriendi in the German Reformation (ISI9-IS28), Aldershot, Ashgate, 2007.

I2. Glissenti, Discorsi morali, ouvr. cité, f. $22 v, \mathrm{I} 07 r, \mathrm{I} 27 r, \mathrm{I} 35 v, \mathrm{I} 38 v, 245 v$. 
qui avait eu une vaste circulation en Europe durant le seizième siècle ${ }^{13}$. À côté de ces images qui mettent en scène le dialogue entre le Philosophe et le Courtisan, d'autres incarnent la fonction proprement méditative de l'œuvre, comme au début du chapitre "Che 'l più eccellente studio è la contemplatione della Morte", où l'on voit une mise en scène allégorique de la contemplation de la mort (fig. 4) ${ }^{\mathrm{I}}$.

Par ailleurs, la dimension théâtrale de l'œuvre est explicitée par Glissenti dans sa préface aux Discorsi, où l'œuvre est dite une représentation de la tragédie des actions humaines: "fu parimente il mio pensiero di rappresentarvi innanzi agli occhi come una tragedia delle attioni humane ${ }^{15} »$. Cette similitude est développée afin d'illustrer la division du dialogue en cinq journées, à savoir cinq journées comparables aux cinq actes d'une tragédie :

Per tanto ho diviso questa operetta in cinque dialoghi, o cinque giornate, come in cinqu'atti di tragedia, co quali andiamo componendo nella vita nostra tutto il progresso di lei, dimostrando le passioni, e gli accidenti, ch'ogn'or l'accompagnano ${ }^{16}$.

Après une longue description des cinq étapes qui forment la narration — du refus des plaisirs sensuels jusqu'à la préparation à la mort — la tragédie humaine est synthétisée par l'image du protagoniste, l'Homme, en équilibre instable entre l'Ange et le Démon, ou bien - pour reprendre des termes chers à la philosophie naturelle - entre Raison et Sens (et il faut souligner la précision avec laquelle Glissenti insiste sur cette double lecture, car nous voyons peut-être ici le double souci de l'auteur, médecin intéressé au salut du corps et de l'âme ${ }^{17}$. La vie humaine, finalement, est une tragédie parce que son protagoniste, l'Homme, comprend l'importance de refuser les plaisirs sensuels quand il est trop tard, c'est à dire quand la Mort arrive, selon une intrigue que nous allons également retrouver dans les drames de Glissenti.

13. Pour l'édition originale de la danse macabre de Holbein, voir Les simulachres et historiées faces de la mort, autant élégamment pourtraictes que artificiellement imaginées, Lyon, Trechsel, I538 (en fac-similé dans H. Green, "Les Simulachres et historiées faces de la mort", commonly called "The Dance of Death", Manchester, A. Brothers, I869). La danse macabre apparut bientôt en Italie : Simolachri, historie, e figure de la morte, Venise, Vincenzo Valgrisi, I545. Sur ce cycle iconographique, voir Kurtz, The Dance of Death..., ouvr. cité, p. I96-198.

I4. Glissenti, Discorsi morali, ouvr. cité, f. $7 r$.

I5. Ibid., f. A[I]r

I6. Ibid.

I7. Ibid., f. A2r: "Che si come nella Tragedia si trovano propositioni facili, varie attioni, e fine doloroso [...] cosi io volendo rapresentare questa Tragedia della vita humana, presi soggetto da questo illustre principe de gli animali; e suppongo che l'Huomo stia con gravità a sedere, come dispensator e giudice della sua vita e delle sue attioni, ascoltando quei due Angeli, buono e cattivo, che lo vanno consigliando: overo (come dicono i Naturali) la Ragione, e il Senso." 
Par contre, avant d'approcher les drames, nous devons nous arrêter sur deux aspects des Discorsi qui sont importants pour comprendre la poétique théâtrale de Glissenti et sa notion du «tragique»: tout d'abord la scène du dialogue; ensuite les idées de l'auteur sur le théâtre.

Le deuxième jour du dialogue - intitulé Estisiphilo - commence avec une digression sur le palais Ducal de Venise et sur la place SaintMarc, célébrés comme un théâtre magnifique, véritable image du monde ${ }^{\mathrm{I} 8}$. L'auteur explique que la structure du palais reflète celle de l'univers, du paradis (les appartements du prince) aux enfers (les prisons au sous-sol), à travers le domaine humain de l'apparence et du mensonge. Le monde est décrit comme "giuoco di Fortuna", "gabbia di matti", "trattenimento di sciocchi», et le palais est dit "giuoco a trappola», "scrigno di astutie», "perdimento di cervello", termes qui, communs parmi les auteurs de la Renaissance, donnent une image tout à fait négative du monde ${ }^{\mathrm{r}}$. En revanche, ce qui rend le cas de Glissenti particulier, c'est justement la projection systématique de ces lieux communs sur un cadre dramatique où même la célèbre métaphore du théâtre du monde est prise à la lettre.

Les Discorsi morali peuvent donc être lus comme un "théâtre en papier" où l'usage des métaphores théâtrales (et nous venons ici au deuxième aspect qu'il faut considérer) acquiert une valeur supplémentaire grâce à une réflexion explicite sur le théâtre. À cette réflexion l'auteur consacre une grande partie de la quatrième journée du dialogue. Lors de la rencontre du Philosophe et du Courtisan avec une actrice professionnelle, Glissenti met en scène une conversation qui vise à montrer la nature pernicieuse des arts théâtraux. L'actrice incarne évidemment l'image du théâtre comme mensonge et illusion selon une tradition qui remonte aux Pères de l'Église, et qui avait été reprise au Xvi ${ }^{\mathrm{e}}$ siècle par Charles Borromée ${ }^{20}$. En se référant à l'expérience professionnelle de l'actrice, le philosophe souligne encore la ressemblance du monde à la scène théâtrale : "il Mondo è una scena, et i Recitanti siamo noi mortali tutti ${ }^{21}$ ». Par contre, dans la vie réelle, les hommes - à l'inverse de ce qui est permis aux acteurs — ne

I8. Ibid., f. $60 v$ : "Le quali cose tutte formavano cosi bel theatro, cosi ricca e sontuosa casa, che per esplicare la sua grandezza non trovava cosa a cui assomigliare la potesse meglio che a tutta la sfera del mondo."

19. Ibid., f. 62r. Parmi les auteurs qui ont utilisé ces images, il faut rappeler Antonio Fileremo Fregoso, Baldassar Castiglione, Torquato Tasso, Ludovico Guicciardini, Guido Casoni, Tommaso Campanella. Sur le thème de la fortune, voir le recueil Il tema della fortuna nella letteratura francese e italiana del Rinascimento, Florence, Leo S. Olschki, I990.

20. Voir Ibid., f. $34 \mathrm{I} v$, pour la vignette qui représente le philosophe et l'actrice. En ce qui concerne Charles Borromée, voir au moins le chapitre "Lazione di Carlo Borromeo" dans F. Taviani, La commedia dell'arte e la società barocca, Rome, Bulzoni, I991.

2I. Glissenti, Discorsi morali, ouvr. cité, f. $344 v$. 
peuvent pas répéter leurs actions. Ils doivent donc se comporter bien pour gagner l'accès aux cieux. Cette conversation implique une discussion sur la valeur morale du théâtre profane, laquelle est rejetée par Glissenti, qui considère que le théâtre profane est un genre consacré au divertissement et bâti sur le mensonge. En revanche - et nous trouvons ici la raison d'être des drames de Glissenti —, lorsque le théâtre devient un instrument d'édification morale, il peut être accepté parmi les activités pédagogiques, à la fois comme théâtre à jouer et comme théâtre à lire ${ }^{22}$.

Comme nous l'avons dit, les drames moraux de Glissenti étaient destinés aux orphelines de Grands Hôpitaux Vénitiens où l'auteur travaillait comme médecin, en particulier l'Hôpital des Malades Incurables et celui des Derelitti ${ }^{23}$. En suivant le modèle du théâtre des Jésuites, les représentations visaient, au même temps, à l'édification morale des acteurs (actrices, en ce cas) et du public. Un processus similaire était conçu pour les lecteurs : d'après ce que nous lisons dans les préfaces des éditions (principalement adressées à des femmes nobles et religieuses), la lecture individuelle et la méditation étaient considérées comme la meilleure façon de lire ce genre de textes ${ }^{24}$.

La nature spirituelle des drames de Glissenti se révèle lorsque nous nous arrêtons sur les intrigues, les personnages et - surtout - sur les lieux où l'action des personnages est censée se dérouler : comme dans le genre médiéval des moralités, le protagoniste est souvent l'Homme qui, aidé par la Raison, la Conscience et — surtout - le Libre Arbitre, affronte les tentations de la Chair. Parfois c'est la rencontre de l'Homme avec la Mort qui fournit l'intrigue, tout en poussant le protagoniste et les spectateurs / lecteurs à méditer sur leur destin. Par ailleurs, face à un modèle ancien comme celui de la psychomachie, la fonction proprement méditative de ce théâtre est bien incarnée par la scène — tout à fait intérieure — où l'action se déroule : le théâtre du monde devient, en fait, un véritable théâtre de l'âme. La Ragione sprezzata, par exemple, se déroule sur la scène du

22. Ibid., f. 344v-345r. Sur le théâtre comme instrument d'édification morale, voir G. Zanlonghi, Teatri di formazione. Actio, parola e immagine nella scena gesuitica del Sei-Settecento a Milano, Milan, Vita e Pensiero, 2002.

23. Pour un encadrement des activités culturelles dans les hôpitaux vénitiens, voir Nel regno dei poveri. Arte e storia dei grandi ospedali veneziani in età moderna, I474-I797, études réunies par B. Aikema et D. Meijers, Venise, Arsenale editrice, 1989. Si l'éducation musicale dans les hôpitaux a été l'objet de nombreuses études, le rôle du théâtre doit encore être exploré.

24. Voir, à cet égard, les préfaces à la duchesse Léonore de Mantoue (Glissenti, La ragione sprezzata, ouvr. cité, f. $2 r$-4v), et celle à Madame Contarina Leoni (Glissenti, Il bacio della giustitia e della pace, ouvr. cité, p. 3); mais il faut rappeler que même les Discorsi morali étaient dédiés à une femme, à savoir la sœur de l'auteur, Glissentia Glissenti (pour la longue préface, voir Glissenti, Discorsi morali, ouvr. cité, f. A2r-a4v). 
monde ("la scena è il Mondo»); Il Mercato overo fiera della vita humana développe la même image en introduisant celle du grand palais du Monde, lieu par excellence du marché des vivants : "la scena è dinanzi al palaggio del Mondo nel cui gran cortile si fa la fiera de tutti i viventi». Ailleurs, la dimension intérieure est encore plus explicite : L'Andrio, qui aborde le sujet du Libre Arbitre comme fonction fondamentale des choix humains, se déroule sur le champ du Libre Arbitre ("la scena è il campo del Libero Arbitrio"); pareillement, la scène de la Sarcodinamia, drame consacré à la puissance de la Chair, est la conscience propre à chacun («la scena è la propria consideratione di ciascuno»). Mais c'est l'Androtoo qui doit surtout attirer notre attention, car dans cette pièce la scène est justement la maison du cœur où la psychomachie se déroule : "la scena è la casa del cuore di ciascheduno, dove gli affetti interni ed esterni dell'huomo, gareggiando a diverso fine, si risolvono finalmente dove lo stesso huomo vuole». Nous reconnaissons ici l'imaginaire que nous avions évoqué au tout début à propos des emblèmes chrétiens de Francesco Pona : tout comme dans le Cardiomorphoseos, le théâtre de Glissenti vise à bâtir des espaces intérieurs où les composantes de la psychologie humaine peuvent être littéralement mises en scène. Le public saisit avec ses yeux (et, fait important, avec ses yeux intérieurs) la vraie nature de l'Âme humaine, ainsi que — grâce aux allégories personnifiées - ses fonctions intellectuelles : la Mémoire, l'Arbitre, la Raison. Il s'agit d'une véritable anatomie de l'âme, comme nous pouvons le voir en regardant, entre autres, la liste de personnages tirée de l'Androtoo de Glissenti ${ }^{25}$.

La question qu'il faut poser à ce point porte sur la fonction contemplative de ces textes et sur leur efficacité : la tragédie de la vie humaine aboutit-elle à une véritable purification? Pour répondre, nous reviendrons sur la fonction méditative de ces textes afin de montrer ce qu'ils ont en commun avec la tradition des exercices spirituels, une tradition qui remonte aux écoles philosophiques de l'Antiquité et qui, à travers l'Antiquité tardive et le Moyen Âge, fut reprise par la culture chrétienne ${ }^{26}$. Dans une tradition aussi vaste, nous allons nous limiter à quelques exemples. $\mathrm{Au} \mathrm{XVI}{ }^{\mathrm{e}}$ siècle, les textes médiévaux latins et vulgaires de méditation chrétienne, principalement consacrés à l'examen de conscience, étaient parmi les livres les plus fréquemment imprimés en Italie (il suffit de citer Bernard

25. Glissenti, L’Androtoo, ouvr. cité, f. A $5 v$.

26. Le texte de référence au sujet des origines de la pratique de l'examen de conscience est P. Hadot, La citadelle intérieure : introduction aux Pensées de Marc Aurèle, Paris, Fayard, 1992. 
de Clairvaux, Domenico Cavalca, Jacopo Passavanti ${ }^{27}$ ). Dans ce contexte, il ne faut pas non plus oublier l'influence majeure des Exercices spirituels d'Ignace de Loyola, publiés à Rome en $1548^{28}$. Si, d'un côté, la nature dramatique des Exercices a été soulignée plusieurs fois par l'érudition moderne, le manuel de Loyola n'a pas été analysé, en revanche, de façon systématique comme œuvre fondamentale pour comprendre le développement du théâtre spirituel. Il ne s'agit pas seulement, d'ailleurs, de nous arrêter à des analogies textuelles : les Jésuites ont, rappelons-le, aussi joué un rôle décisif dans le cadre des activités de catéchisme et d'édification qui avaient lieu dans les Hôpitaux Vénitiens (Loyola, en particulier, avait entretenu des liens importants avec les Incurables et les Derelitti, où Glissenti produisit plus tard ses pièces de théâtre).

Deux exemples vont nous aider à mieux comprendre ce point. Nous allons en premier lieu aborder le triomphe de la Mort dans la scène finale de la tragédie La Ragione Sprezzata. Une grande danse macabre se déroule dans le "théâtre» des Parques : hommes et femmes, acteurs sur la scène du monde, sont condamnés aux flammes des enfers pour leurs péchés. La personnification de la Nature, qui arrive trop tard pour les sauver, se plaint du sort de ses enfants (selon le modèle célèbre des Lamentations de la Nature d'Alain de Lille). Les réjouissances des plaisirs humains sont remplacées par un silence qui domine le vaste théâtre du monde ${ }^{29}$. S'ensuit alors une description frappante des châtiments infernaux visant à inspirer au public le repentir à travers l'examen de conscience : "consideratione delle proprie colpe», comme Glissenti le dit dans la préface de la pièce, où il s'arrête sur les effets produits chez les spectateurs lors de sa première représentation à l'Hôpital des Incurables. La préface décrit la réaction du public comme une véritable expérience de purification tout en exploitant le lexique typique des discussions contemporaines sur le genre tragique : "Ma parlando di quella utilità che deve essere propria di simili favole, che è del purgar gli animi da $i$ vitii et invitarli alla virtù, certamente che utilissima è riuscita.»Ce qui montre, par ailleurs, encore mieux

27. Pour un encadrement de la tradition littéraire italienne sur la pratique de la méditation chrétienne, voir I. Gallinaro, I castelli dell'anima: architetture della ragione e del cuore nella letteratura italiana, Florence, Leo S. Olschki, I999.

28. I. de Loyola, Exercitia Spiritualia, Rome, Antonio Blado, I548. Dans la vaste bibliographie sur les Exercices de Saint Ignace, voir au moins l'étude fondamentale de P.-A. Fabre, Ignace de Loyola. Le lieu de l'image. Le problème de la composition de lieu dans les pratiques spirituelles et artistiques jésuites de la seconde moitié du XVI $I^{e}$ siècle, Paris, Vrin-EHESS, 1992.

29. Glissenti, La ragione sprezzata, ouvr. cité, acte 4, scène I9, f. I84r-v: "Ma qual silentio inusitato e novo, / taciturno fia il mondo e cheto il tutto? I Che dove pria di genti il gran susurro, / de' godenti le feste, i suoni, e $i$ cantil facean d'intorno l'aria tintinnire, / hor par che nulla s'oda e taccia il mondo?" 
le but du théâtre de Glissenti — et qui nous rappelle ce que nous avons dit à propos du dialogue entre le philosophe et l'actrice dans les Discorsi morali —, c'est la comparaison explicite avec l'inefficacité des prêcheurs. Ce que les mots éloquents des prêcheurs n'ont pas produit - c'est-à-dire le repentir du public — a été obtenu par la pièce théâtrale :

[...] poi che ha spaventato di sì fatta maniera molti, et altri posti in sì fatta consideratione de i fatti loro, che quello, che non hanno fatto molte e frequentate persuasioni di eloquenti Predicatori, et le rigide ammonitioni di Prelati, ha fatto la presente rappresentatione, col porli innanti a gli occhi l'essempio del fine loro; che mossi più dall'essempio che dalle parole, si sono rissoluti a confessarsi, e chiamarsi in colpa di molti eccessi loro, che per più anni se n'erano passati prima senza alcuna consideratione, o rissentimento delle sue colpe ${ }^{30}$.

En d'autres termes, l'exemple mis en scène par la pièce a le pouvoir de réformer les spectateurs, qui, grâce à la contemplation des châtiments infernaux, sont amenés à examiner leur conscience et à se repentir.

À cet égard, nous pouvons rajouter que la contemplation de l'Enfer dans la scène finale de La Ragione sprezzata suit une structure que l'on retrouve chez Ignace de Loyola :

Attendan tutti mentre ancor son vivil a dispensar sua vita in opre sante, / a seguir la Ragion, fuggir li Sensi, I e disprezzar ogni mondan diletto, I che li condanna a sempiterne pene. I Mirino al Tempo, che lor rubba gli anni./ Si ricordin di noi [les Parques], che le lor tele/ sollecite tessiam. Te teman figlia [la Mort], I che rigida a nissun giamai perdoni. I E si spaventin del tremendo giornol che giudicar li dee, con tal essempio / di tanti, e tanti a sempiterni horrori, / per fugaci piacer dannati sempre ${ }^{35}$.

Dans le cinquième des Exercices Spirituels Ignace recommande de commencer par la compositio, véritable arrangement de la scène, pour poursuivre ensuite avec la vision proprement dite ("videre visu imaginationis»). $\grave{A}$ travers les cinq sens spirituels (spectare, audire, persentire, gustare, tangere), le contemplatif est en gré de saisir les châtiments et stimuler ce qu'il appelle «le ver de la conscience», reprenant ici une image efficace que Loyola tire du traité De interiore domo longtemps attribué à Bernard de Clairvaux ${ }^{32}$.

3o. Ibid., f. A7r-v.

31. Ibid., acte 5 , scène I8, f. I83r-v.

32. I. de Loyola, Exercitia Spiritualia, ouvr. cité, I, 5 : «Primum praeambulum, compositio, quae hic est, videre visu imaginationis longitudinem, latitudinem, et profunditatem inferni. [...] Punctum primum est, spectare per imaginationem vasta inferorum incendia, et animas, igneis quibusdam corporibus, velut ergastulis, inclusas. Secundum, audire imaginarie planctus, ejulatus, vociferationes atque blasphemias in Christum et sanctos ejus illinc erumpentes. Tertium, imaginario etiam olfactu fumum, sulphur, et sentinae cujusdam seu faecis, atque putredinis graveolentiam persentire. Quartum, gustare similiter res amarissimas, ut lachrymas, rancorem, conscientiaeque vermen. Quintum, tangere quodammodo ignes illos, quorum tactu animae ipsae amburuntur." 
La centralité de la pratique de l'examen de conscience dans la poétique théâtrale de Glissenti est confirmée par son élaboration purement dramatique, comme nous le voyons dans L'Androtoo. Sur la scène du cœur, deux personnifications allégoriques - la Conscience et le Remords représentent une véritable discipline morale. La Conscience, en parlant d'elle-même, dit :

O come facilmente l'huomo cade da l'innocenza sua, da la bontate, quando non ha rimorso che lo rodi, né conscienza che lo punga o fieda,

et le Sens, de son côté :

Questa co 'l suo rimorso l'huom sì assale,

si lo rode e lo lacera sempre

ch'ei timido sarretra, quanto io innanzi

lo vo guidando del peccato in grembo ${ }^{33}$.

La Conscience est donc assiégée par les Sens, tout comme dans un autre emblème de Pona, où le cœur est représenté comme une tour prise d'assaut par les démons, selon un topos architectural qui remonte au livre de Jérémie et qui eut une diffusion considérable au Moyen Âge. Ici aussi il faut que nous nous contentions d'un seul exemple, à savoir une citation tirée des Méditations pieuses de Bernard de Clairvaux où l'on trouve l'image de la conscience assiégée par les Sentiments ${ }^{34}$. Cette tradition, bien évidemment, est la même que l'on retrouve chez Ignace. La scène intérieure des exercices ignaciens, d'ailleurs, n'est pas différente du lieu où se déroule l'action dramatique de la pièce de Glissenti et le processus de purification envisagée par la Conscience elle-même semble suivre de près le vadémécum d'Ignace. Il suffit de comparer deux passages pour saisir ce point :

Esaminate ben l'interno vostro, se in parole, se 'n fatti, se con l'opre, se col consenso, o col pensier vagante

33. Glissenti, L'Androtoo, ouvr. cité, acte 5, scène 7, p. 166-167.

34. Bernard de Clairvaux, Meditationes, XII (Patrologia Latina, I84, 503b-d) : "Adjuva me, Domine Deus meus, quoniam inimici mei animam meam circumdederunt: corpus scilicet, mundus, et diabolus. A corpore fugere non possum, nec ipsum a me fugare. [...]. Mundus vero circumcingit et obsidet me undique, et per quinque portas, videlicet per quinque corporis sensus, scilicet visum, auditum, gustum, odoratum et tactum, sagittis suis me vulnerat; et mors intrat per fenestras meas in animam meam. [...] Porro diabolus quem videre non possum [...] tetendit arcum suum, et in eo paravit sagittas suas, ut vulneret me repente." 
sete transcorso in qualche grave errore,

acciò tantosto penitenza segua ${ }^{35}$.

Punctum primum sit processus quidam, per quem peccata totius vitae in memoriam revocantur, percursis gradatim discussisque annis, et spatiis temporum singulis. [...] Secundum est peccata ipsa perpendere [...] Tertium est, considerare me ipsum quisnam aut qualis $\operatorname{sim}^{36}$.

Par ailleurs, le lien de la production dramatique de Glissenti avec la tradition jésuite et les textes dévotionnels devient explicite lors du curieux échange entre Conscience et Mémoire dans le deuxième acte de l'Androtoo. Le dialogue, bâti à un rythme très soutenu, porte sur un livre que la Mémoire doit acheter. Ayant oublié le titre, le personnage est aidé par la Conscience, qui se montre tout à fait experte dans le domaine des ouvrages de dévotion chrétienne. Parmi le titres suggérés à son interlocutrice, l'on retrouve, pas par hasard, La dottrina christiana, le Modo d'essaminar la conscienza et, justement, le Modo di confessarsi degnamente ${ }^{37}$.

Finalement, la tragédie de la vie humaine — approchée par Glissenti au double niveau du code métaphorique et du code théâtral proprement dit - se développe dans un projet d'édification morale bien cohérent, lequel vise à une forme de purification qui, assez différente de la catharsis aristotélicienne, semble trouver son espace idéal ainsi que son aboutissement dans la pratique de la méditation.

N.D.A. - Nous remercions Maude Vanhaelen et Anna Pegoretti pour leurs suggestions.

\section{Bibliographie}

Bernheimer Richard, "Theatrum mundi", Art Bulletin, n 38, 1956, p. $225-247$.

Buccini Stefania, Francesco Pona: l'ozio lecito della scrittura, Florence, Leo S. Olschki, 2013.

Christian Lynda, Theatrum Mundi: The History of an Idea, New YorkLondres, Garland, 1987.

Costanzo Mario, Il Gran Theatro del Mondo, Milan, Scheiwiller, 1964. Curtius Ernst Robert, Letteratura europea e Medio Evo Latino, Florence, La Nuova Italia, I995.

35. Glissenti, L'Androtoo, ouvr. cité, acte 2, scène 8, p. 58-59.

36. Loyola, Exercitia spiritualia, ouvr. cité, I, 2.

37. Glissenti, L'Androtoo, ouvr. cité, acte 2, scène Io, p. 64-65. 
Fabre Pierre-Antoine, Ignace de Loyola. Le lieu de l'image. Le problème de la composition de lieu dans les pratiques spirituelles et artistiques jésuites de la seconde moitié du XVI siècle, Paris, Vrin-EHESS, I992.

Gallinaro Ilaria, I castelli dell'anima: architetture della ragione e del cuore nella letteratura italiana, Florence, Leo S. Olschki, 1999.

Glissenti Fabio, In quinque praedicabilia Porphyrij. In sex principia Gilberti Porretani. In Praedicamenta Aristotelis. In perihermenias Aristotelis. In priora, et posteriora Aristotelis. Per methodicas Divisiones brevissima commentaria, Venise, Giovan Battista Ciotti, I594.

—, Discorsi morali dell'eccellente signor Fabio Glissenti. Contra il dispiacer del morire. Detto Athanatophilia, Venise, Domenico Farri, I596.

—, La Ragione sprezzata, Serravalle, Marco Claseri, I606.

—, Il bacio della Giustitia e della Pace, Venise, Giovanni Alberti, I607.

—, L'Andrio cioè l'Huomo virile, Venise, Giovanni Alberti, I607.

—, Il Diligente overo il Sollecito, Venise, Giovanni Alberti, I608.

-, La Morte inamorata, Venise, Giovanni Alberti, I608.

—, L'Androtoo cioè l'Huomo innocente, Venise, Marco Ginammi, I6ı6.

—, La giusta Morte, Venise, Marco Ginammi, I6r7.

—, Lo Spensierato fatto pensoroso, Venise, Marco Ginammi, I6I7.

- Il mercato overo la fiera della vita humana, Venise, Marco Ginammi, I620.

—, La Sarcodinamia cioè la possanza della carne, Venise, Marco Ginammi, I620.

Hadoт Pierre, La citadelle intérieure : introduction aux Pensées de Marc Aurèle, Paris, Fayard, 1992.

Il tema della fortuna nella letteratura francese e italiana del Rinascimento, Florence, Leo S. Olschki, I990.

KurTz Léonard P., The Dance of Death and the Macabre Spirit in European Literature, New York, Columbia University, 1934.

Les simulachres et historiées faces de la mort, autant élégamment pourtraictes que artificiellement imaginées, Lyon, Trechsel, I538.

Loyola Ignace de, Exercitia Spiritualia, Rome, Antonio Blado, I548.

McClure George, "The Artes and the Ars moriendi in Late Renaissance Venice: The Professions in Fabio Glissenti's Discorsi morali contra il dispiacer del morire, detto Athanatophilia (1596)", Renaissance Quarterly, $\mathrm{n}^{\circ}$ 51, 1998, p. 92-I27.

Nel regno dei poveri. Arte e storia dei grandi ospedali veneziani in età moderna, I474-I797, études réunies par B. Aikema et D. Meijers, Venise, Arsenale editrice, 1989. 
Pona Francesco, Cardiomorphoseos sive ex corde desumpta emblemata sacra, Vérone, Bartolomeo Merlo, I645.

Piantoni Luca, "Morte a Venezia. L'Athanatophilia di Fabio Glissenti, I596", dans Visibile teologia. Il libro sacro figurato in Italia tra Cinque e Seicento, études réunies par E. Ardissino et E. Selmi, Rome, Edizioni di Storia e Letteratura, 20I2, p. 22I-250.

Reinis Austra, Reforming the Art of Dying: The Ars moriendi in the German Reformation (ISID-I528), Aldershot, Ashgate, 2007.

SAso Anna Laura, "Glissenti, Fabio", Dizionario Biografico degli Italiani, $\mathrm{n}^{\mathrm{o}}$ 57, 200I, p. 406-408.

Simolachri, historie, e figure de la morte, Venise, Vincenzo Valgrisi, I545.

Taviani Ferdinando, La commedia dell'arte e la società barocca, Rome, Bulzoni, I99I.

Tenenti Alberto, Il senso della morte e l'amore della vita nel Rinascimento, Turin, Einaudi, 1977.

Yates Frances Amelia, Theatre of the World, Londres, Routledge \& Kegan Paul, 1969.

Zanlonghi Giovanna, Teatri di formazione. Actio, parola e immagine nella scena gesuitica del Sei-Settecento a Milano, Milan, Vita e Pensiero, 2002. 


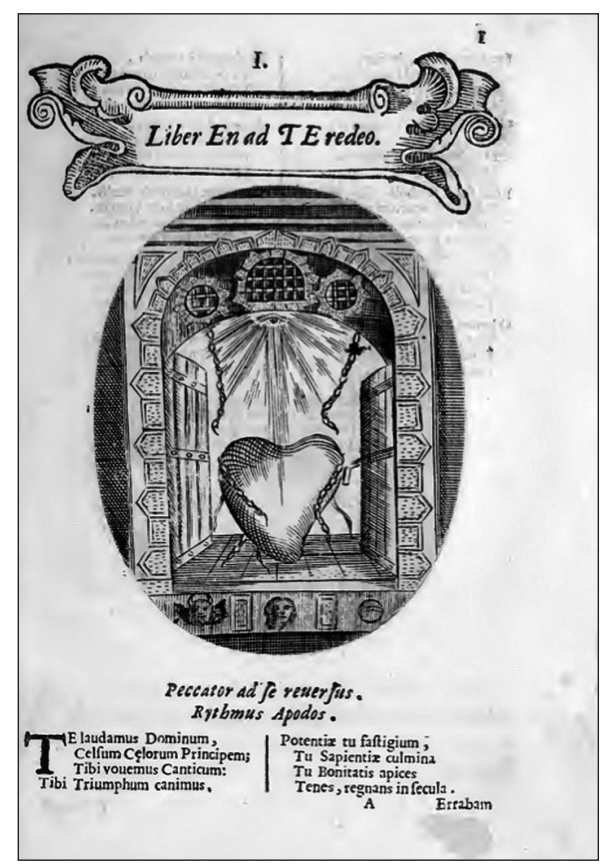

Fig. I. - Francesco Pona, Cardiomorphoseos, Vérone, Bartolomeo Merlo, I645, p. I.

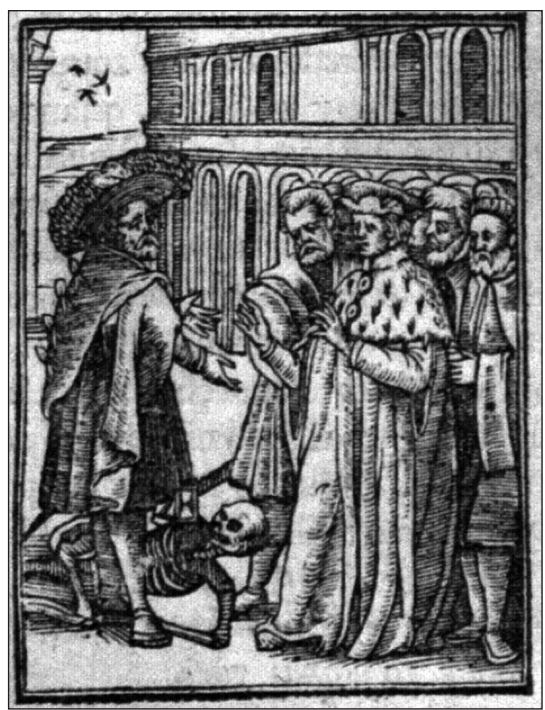

Fig. 3. - Fabio Glissenti, Discorsi morali, Venise, Domenico Farri, I596, f. $22 v$.

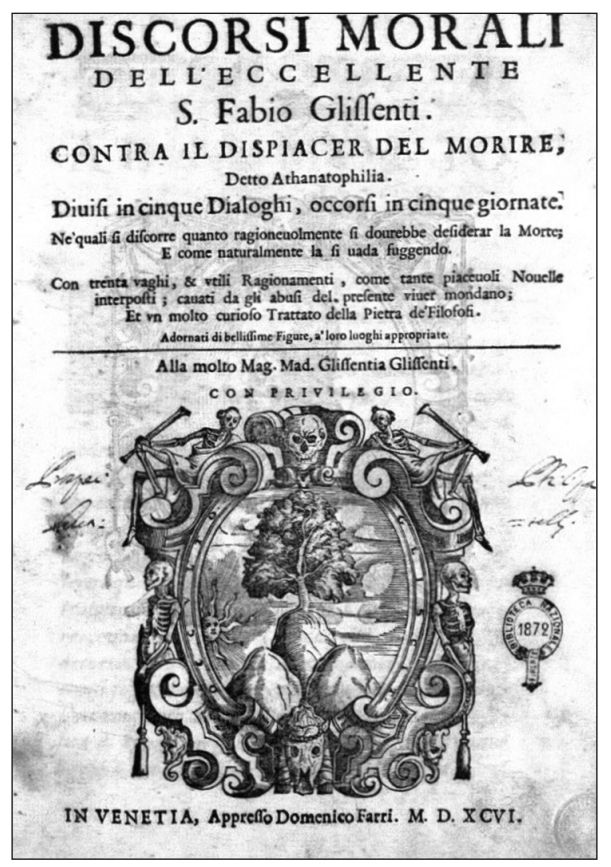

Fig. 2. - Fabio Glissenti, Discorsi morali, Venise, Domenico Farri, I596, frontispice.

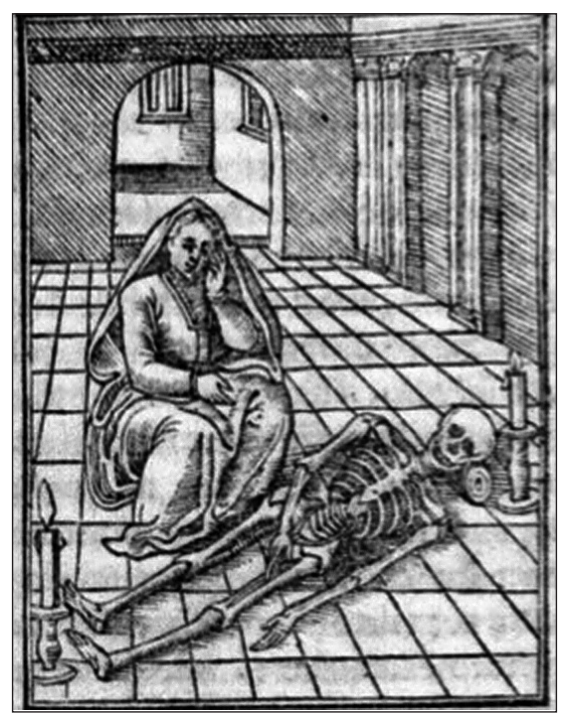

Fig. 4. - Fabio Glissenti, Discorsi morali, Venise, Domenico Farri, I596, f. $7 r$. 